\title{
Specificity of sites within eight-membered ring zeolite channels for the carbonylation of methyls to acetyls
}

\author{
Aditya Bhan, Ayman D. Allian, Glenn J. Sunley, David J. Law and \\ Enrique Iglesia \\ Supporting Information
}

\section{Computational Methods}

The hydroxyl band in Mordenite (MOR) is asymmetric because of different stretching frequencies for hydroxyls within 8-member (8-MR) and 12-member (12-MR) channels. The main challenges in deconvoluting these overlapping bands are to determine (i) the number of bands and their frequency and (ii) the appropriate function to describe the shape of these bands (e.g., Lorentzian, Gaussian or combinations thereof). For illustration purposes, we describe these numerical methods in the specific context of their use to determine $\mathrm{H}^{+}$location and concentration within acid and cation-exchanged forms of MOR.

\subsection{Peak-finding Method}

Second derivative analysis is the most widely used strategy to locate the center of infrared bands, but this technique can be misleading and difficult to apply specially with the ubiquitous noise present in typical infrared spectra. Here, the peak center position is identified by examination of the right singular vector, shown in Figure S.1., obtained using Singular Value Decomposition (SVD) methods ${ }^{1}$. 


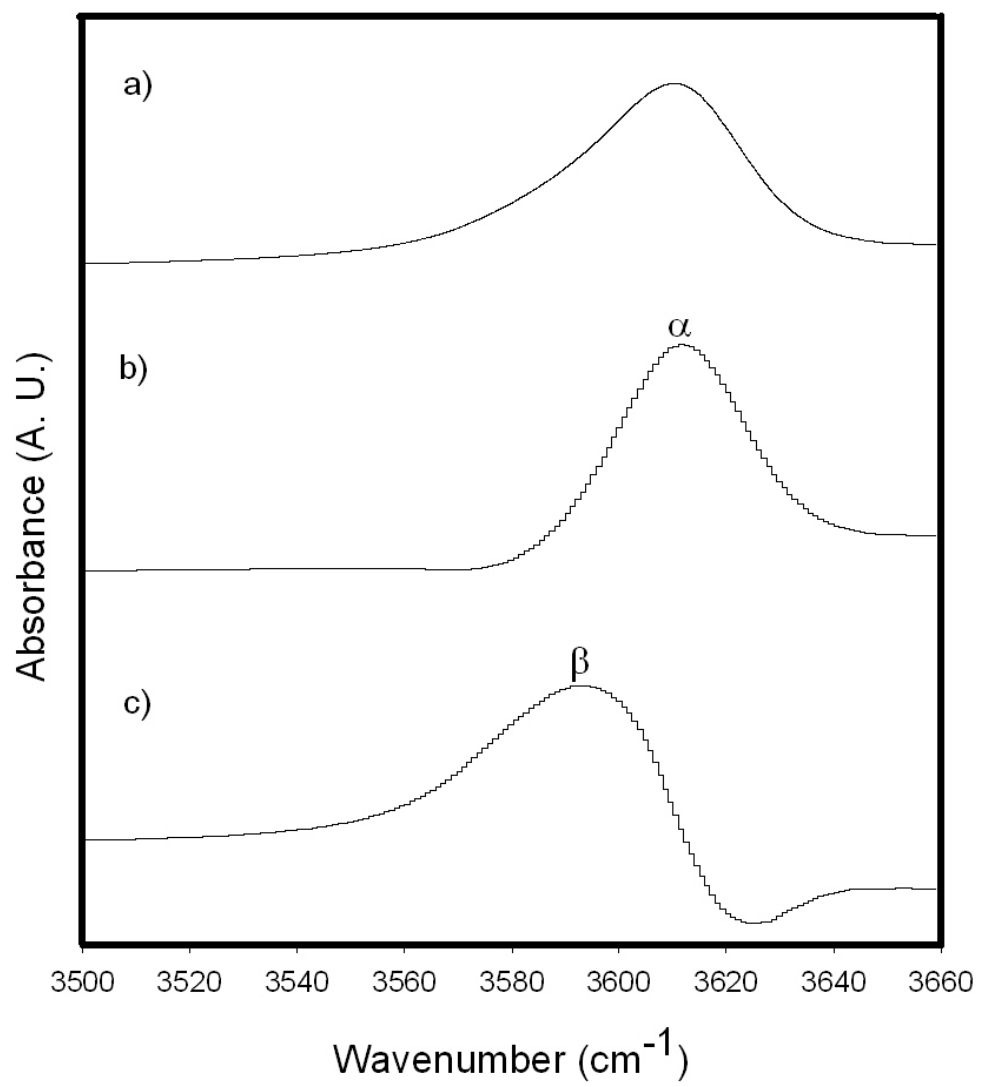

Figure S.1 The (a) first, (b) second and (c) third right singular vectors from the SVD of the experimental data $(\mathrm{H}-\mathrm{MOR}$ sample; $\mathrm{Si} / \mathrm{Al}=10$, Zeolyst $)$.

SVD enumerates the vectors in the order of their singular values, which represent their contribution to the total variance in the recorded spectra. Thus, the first right singular vector represents the average of all measured spectra, while latter right singular vectors reflect the pure components present within them. The features of each pure component present can be deconvoluted when many spectra are collected and the relative spectral contributions from each species vary significantly among these spectra. Indeed, two new resolved bands, $\alpha$ and $\beta$, at 3592 and $3611 \mathrm{~cm}^{-1}$ are apparent (see Figure S.1) in the second and third right singular vectors, respectively, upon SVD of infrared spectra of MOR samples with varying $\mathrm{Na}^{+}$content $\left(\mathrm{Na}^{+}\right.$cations preferentially exchange $\mathrm{H}^{+}$within 8-MR channels of MOR). The position of these two bands are in excellent agreement 
with infrared studies using probe molecules that selectively titrate $\mathrm{OH}$ groups in 8-MR $\left(3592 \mathrm{~cm}^{-1}\right)$ and 12-MR channels $\left(3611 \mathrm{~cm}^{-1}\right)$ of MOR ${ }^{2-4}$.

\subsection{Curve-fitting of experimental infrared spectra}

The Pearson VII model ${ }^{5}$ was selected to describe infrared bands because it requires no priori assumptions about shape and only three parameters $(a, p$, and $q)$ along with the peak center position $v^{o}$ are required, as shown in equation S.1. The Pearson VII model is more general than either the Lorentzian or Gaussian functions ${ }^{6}$ due to the flexibility in the value of $q$, known as the Pearson parameter. When $q$ approaches 1 , the Pearson VII model resembles a Lorentzian in character and as $q$ approaches infinity, it becomes Gaussian. The values of these parameters were obtained via optimization methods that minimize the difference between curve-fitted and the experimental spectra.

$$
A_{i}=\frac{a}{p\left(1+\frac{\left(v_{i}-v^{o}\right)^{2}\left(2^{1 / q}-1\right)}{p}\right)^{q}}
$$

A similar approach was proposed for the analysis of spectra collected during insitu monitoring of homogenous catalysts ${ }^{7}$. This previous study used Simplex optimization methods. Here, we use simulated annealing methods because of their robustness in locating global error minima in estimating parameters for equation S.1. The estimated pure component bands for O-H groups in 8-MR and 12-MR channels, and the curve-fit obtained using the methods outlined above are shown in Figure S.2 for the H$\operatorname{MOR}\left(\mathrm{H}_{100} \mathrm{Na}_{0} \mathrm{MOR}\right)$ sample. 


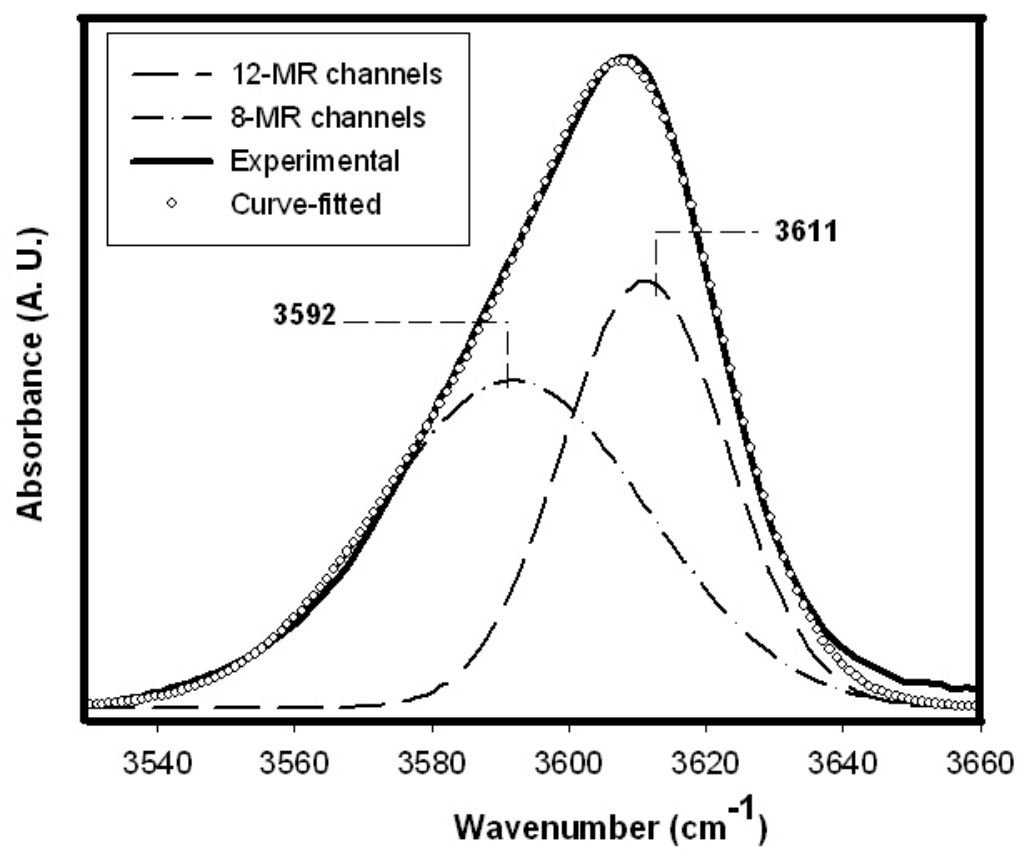

Figure S.2 Comparison of the experimental (solid curve) with the curve-fitted spectrum along with estimated pure component spectra. The experimental data are shown for the H-MOR $\left(\mathrm{H}_{100} \mathrm{Na}_{0} \mathrm{MOR}\right)$ sample.

Figure S.2 shows that the curve-fitted spectrum is in excellent agreement with the measured spectrum except for a slight mismatch in the $3640-3660 \mathrm{~cm}^{-1}$ region, which is likely to reflect slight inaccuracies in background subtraction. The estimated band for the $\mathrm{O}-\mathrm{H}$ vibration in the 8 -MR channels centered at $3592 \mathrm{~cm}^{-1}$ was broader than the one estimated for the main 12-MR channels, consistent with previous literature reports ${ }^{4}$.

\subsection{Site concentration profiles}

The intensities of the pure component spectra $\hat{a}_{2 \times 166}$ for $\mathrm{O}-\mathrm{H}$ vibrations in the 12MR and 8-MR channels were normalized to unity before estimating their relative contributions; their molar extinction coefficients were assumed to be similar. The experimental shifts in band centers among experiments, discussed by Makarova et al. ${ }^{4}$, led us to allow the peak center position to move $v^{o} \pm x$ among spectra. The pure component spectra obtained by optimization of the $[a, p, q]$ parameters in equation S.1 
were shifted by $x$ wavenumbers $\left( \pm 5 \mathrm{~cm}^{-1}\right)$. This assumes that band shape is unaffected by this shift. The optimal values of $x$ were estimated using the following procedure. First, an estimate of the relative concentration, $\hat{c}_{5 x 2}$, was obtained from equation S.2.

$$
\hat{c}_{5 \times 2}=A_{5 x 166}\left[\hat{a}_{2 \times 166}\right]^{+}
$$

in which $\left[\hat{a}_{2 \times 166}\right]^{+}$is the pseudo-inverse of the estimated pure component spectra.

Second, the calculated spectra $\hat{\mathbf{A}}_{5 \times 166}$ was evaluated from equation S.3.

$$
\hat{\mathbf{A}}_{5 \times 166}=\hat{c}_{5 \times 2} \quad \hat{a}_{2 \times 166}
$$

Finally, the pure component spectra were shifted using these values of $x$ to achieve a minimum difference between experimental and calculated spectra, $\hat{\mathbf{A}}_{5 \times 166}-A_{5 x 166}$. Figure S.3 shows the estimated pure component spectra, normalized to unity after allowing this shift in the peak center.

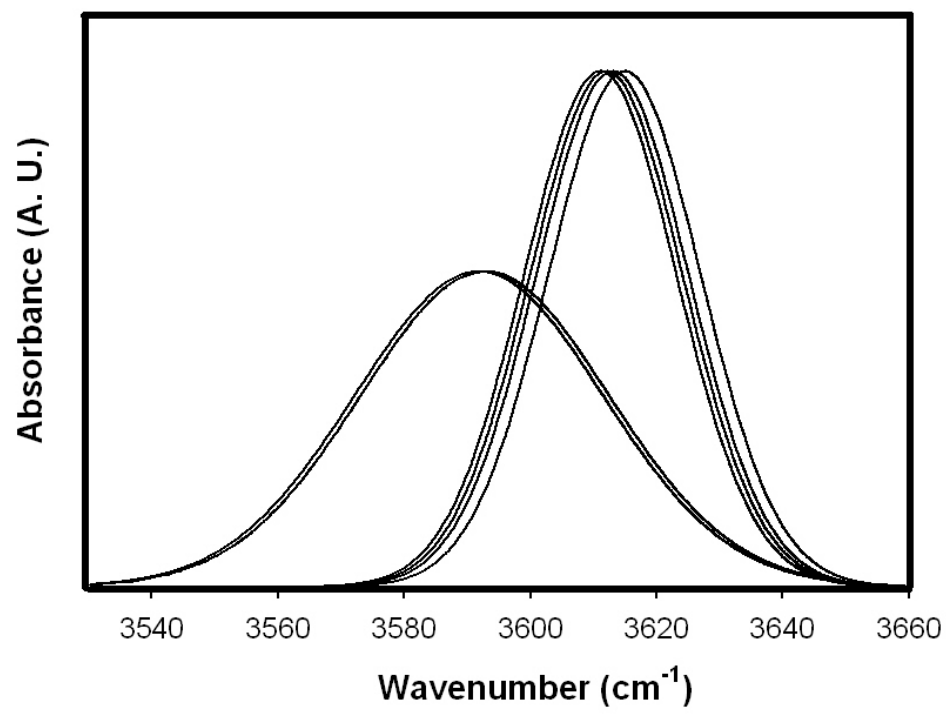

Figure S.3 The estimated pure component spectra for the high frequency $\left(3610 \mathrm{~cm}^{-1}\right)$ and low frequency $\left(3590 \mathrm{~cm}^{-1}\right)$ bands in their normalized form. 
Figure S.3 indicates that the center of the band for O-H groups within 12-MR channels varied more among spectra than for the lower frequency band (O-H groups in 8-MR channels), as also reported by Makarova et al ${ }^{4}$. The optimal pure components shown in Figure S.3 were used to estimate the relative numbers of protons in 8-MR and 12-MR channels.

\subsection{Proton distribution in $\mathrm{H}-\mathrm{MOR}$}

In addition to titration studies using base probe molecules (n-hexane, pyridine and 2,6-dimethyl pyridine) discussed in the manuscript, four replicate samples of the $\mathrm{H}_{100} \mathrm{Na}_{0} \mathrm{MOR}$ sample were examined by this band deconvolution analysis to determine the accuracy and reproducibility of the proton distributions obtained. Figure S.4 shows $\mathrm{H}^{+}$concentrations in the $\mathrm{H}_{100} \mathrm{Na}_{0} \mathrm{MOR}$ sample based on probe molecule titration studies discussed and these replicate measurements. These independent studies predict 55-60\% $\mathrm{H}^{+}$occupancy in 8-MR channels and show the consistency and robustness of the band deconvolution procedure used to determine them.

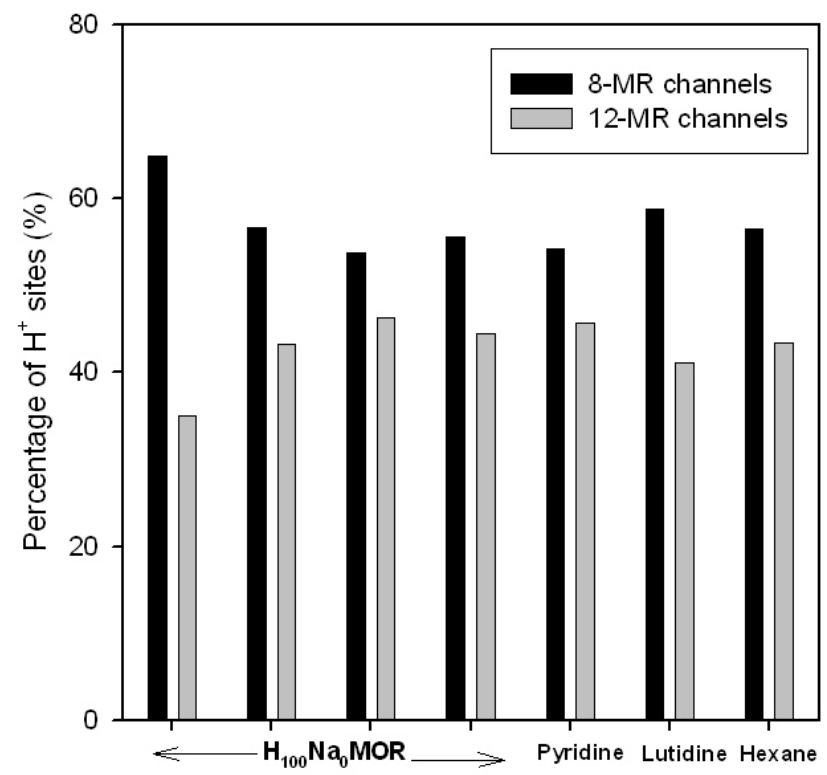

Figure S.4 The percentage of $\mathrm{H}^{+}$sites in the 8-MR and 12-MR channels of $\mathrm{H}_{100} \mathrm{Na}_{0} \mathrm{MOR}$ determined by band deconvolution analysis of the hydroxyl region. 
Recently, Marie et al. ${ }^{8}$ have reported a third $v(\mathrm{OH})$ component at $3605 \mathrm{~cm}^{-1}$ observed at intermediate levels of Na-exchange, and have assigned this band to a hydroxyl species located at the opening window between the main channels and the side pockets located at the $\mathrm{O} 2$ framework oxygen site in MOR. Our infrared studies and band deconvolution protocols have only dealt with two different locations of $\mathrm{OH}$ groups. Although we cannot rule out the possibility of a small fraction of $\mathrm{H}^{+}$that inter-change between 8-MR and 12MR channels, we believe that it is unlikely that we would get such consistency in $\mathrm{H}^{+}$ concentrations in $\mathrm{MOR}$ from $\mathrm{OH}$ band deconvolution and probe molecule titration studies (Figure S4) if a significant fraction of $\mathrm{H}^{+}$were to move between the channel systems.

\section{References.}

1. Malinowski, E., Factor Analysis in Chemistry. $2^{\text {nd }}$ ed.; John Wiley and Sons, Inc.: New York, 2002.

2. $\quad$ Maache, M.; Janin, A.; Lavalley, J. C.; Benazzi, E. Zeolites 1995, 15, (6), 507.

3. $\quad$ Datka, J.; Gil, B.; Kubacka, A. Zeolites 1997, 18, (4), 245.

4. Makarova, M. A.; Wilson, A. E.; van Liemt, B. J.; Mesters, C.; de Winter, A. W.; Williams, C. Journal of Catalysis 1997, 172, (1), 170.

5. $\quad$ Elderton, W. P.; Johnson, N. L., Systems of Frequency Curves. Cambridge University Press: 1969; p 224.

6. $\quad$ Michette, A. G.; Pfauntsch, S. J. Journal of Physics D-Applied Physics 2000, 33, (10), 1186.

7. $\quad$ Chen, L.; Garland, M. Applied Spectroscopy 2003, 57, (3), 331.

8. Marie, O.; Massiani, P.; Thibault-Starzyk, F. J. Phys. Chem. B 2004, 108, (16), 5073. 